\title{
446 - Persistent Delusional Disorder (Late Paraphrenia) - An innovative and cost effective clinical model in the community by older adult's mental health crisis and home treatment team.
}

\section{Authors}

Dr Sabarigirivasan Muthukrishnan MRCPsych, Consultant Old Age Psychiatrist and Principal Investigator of Kingshill Research Centre; Dr Kate Hydon, Specialty Doctor and Cristie Howells, Band 7 Clinical Lead.

\section{Aims}

To review the various available clinical models of care delivery for patients with persistent delusional disorder (PDD) in community and economically evaluate the REACT model of safe care delivery- REACT's Assertive and Prudent- Model of Safe Care ( RAP-MoSC).

\section{Methods}

REACT (Response Enhanced Assessment Crisis and Home Treatment Team) is the only bespoke crisis and home treatment team for older adults with mental health problems in the whole of Wales available only for the residents of Cardiff and Vale of Glamorgan through Cardiff and Vale University Health Board..

It was set up on 28 February 2012. The cases of PDD in REACT service since its inception to 31 Dec 2016 were studied in relation to the assertive and prudent health care model. The economic evaluation of this service model for PDD patients was studied in detail.

\section{Results of the study}

The RAP-MoSC model is economically effective in avoiding patients getting admitted to hospital under Mental Health Act by managing them safely in the community.

During the period between 28 February 2012 and 31 December 2016 there were 44 patients with a diagnosis of PDD in REACT's case load. Only 3 patients got admitted to mental health assessment ward with the average length of stay period of 120 days. 41 patients were safely managed in the community with REACT with an average length of stay period of 21 days in REACT.

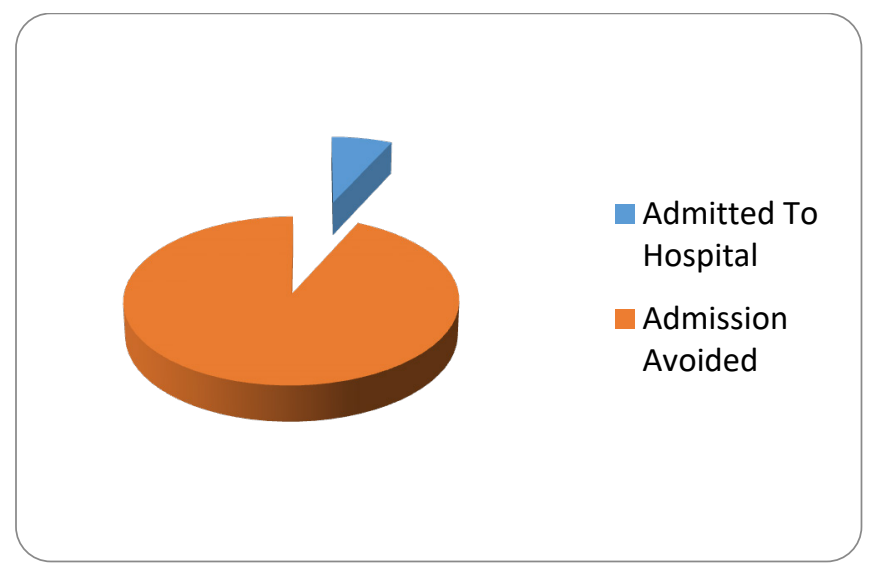

A PDD patient will cost NHS $£ 21,000$ if admitted to a mental health bed. If the patient is managed in the community with RAP-MoSC model of care the cost will be $f 1533$. 


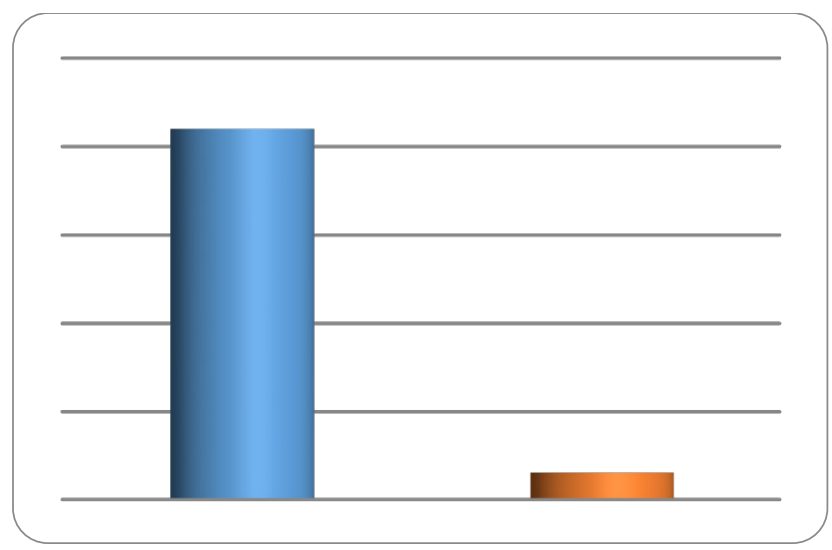

REACT saved $£ 793,548$ by avoiding 41 PDD patients being admitted into hospital during an episode of delusional intensification in the period February 2012 to December 2016. PDD patients need under the RAP-MoSC model a bespoke MDT approach with better communication between secondary mental health and primary care services. Assertive and Prudent Clinical leadership is needed to sustain the RAPMoSC in the community. Clinical reflections of this model of care will be presented in the conference.

\section{Conclusions}

On reflection REACT found that the key points in working with PDD are;

- Using a 'foot in the door approach'

- Mental health professionals introducing themselves as Health professionals

- Remote prescribing 